\title{
POINTWISE ERGODIC THEOREMS
}

\author{
BY \\ M. A. AKCOGLU
}

Introduction. The purpose of this paper is to extend various known results in operator ergodic theory to give a direct approach to pointwise ergodic theorems. The main step in this approach is a maximal ergodic theorem (Theorem 1), which is a generalization of the result given in [1]. A corollary (Theorem 2) of this theorem is Chacon's ergodic theorem [6] for positive operators which contains both Birkhoff's ergodic theorem [2] (or, more generally, Dunford-Schwartz's theorem [9] for positive contractions) and Chacon-Ornstein's ratio ergodic theorem [4]. Theorem 1 is also used to obtain the identification of the limit in a straightforward way. In the final part of the paper Theorem 2 is generalized to nonpositive operators to give a direct proof of Chacon's general ergodic theorem [6].

Definitions and basic lemmas. Let $(X, \mathscr{F}, \mu)$ be a $\sigma$-finite measure space and $L_{1}=L_{1}(X, \mathscr{F}, \mu)$ the Banach space of equivalence classes of integrable complex valued functions on $X$. The elements of $L_{1}$ will be identified by their representative functions and the relations between them will be considered holding up to sets of zero measure. $L_{1}^{+}$is the positive cone of $L_{1}$, consisting of nonnegative $L_{1}$-functions. All subsets of $X$ considered in this paper are measurable either by assumption or construction.

Let $T$ be a contraction on $L_{1}$, i.e., a linear operator $T: L_{1} \rightarrow L_{1}$ for which $\|T\| \leqq 1$ with the usual definition of the norm of an operator on a Banach space. We denote by $\tau$ a positive contraction on $L_{1}$, i.e., a contraction such that $\tau L_{1}^{+} \subset L_{1}^{+}$.

If $F=\left\{f_{0}, f_{1}, f_{2}, \ldots\right\}$ is a sequence of complex valued functions on $X$ and $\alpha$ is a complex number then $\alpha F=\left\{\alpha f_{0}, \alpha f_{1}, \alpha f_{2}, \ldots\right\}, S_{n} F=\sum_{k=0}^{n} f_{k}, n \geqq 0$ and $S_{\infty} F=\sum_{k=0}^{\infty} f_{k}$ (a.e.), if it exists. For an $L_{1}$-function $f$, let $f_{T}=\left\{f, T f, T^{2} f, \ldots\right\}$ and $S_{n}(f, T)=S_{n} f_{T}$.

Definition 1. A sequence $P=\left\{p_{0}, p_{1}, p_{2}, \ldots\right\}$ of nonnegative, finite and measurable functions on $X$ is called $T$-admissible (or, simply, admissible) if $f \in L_{1}$, $n \geqq 0$ and $|f| \leqq p_{n}$ imply that $|T f| \leqq p_{n+1}$.

Admissible sequences have been introduced by Chacon [6], to give a mutual generalization (Theorem 6 of the present paper) of the Chacon-Ornstein theorem [4] and the Hopf-Dunford-Schwartz theorem [9]. Note that if $f \in L_{1}^{+}$then $f_{\tau}=\left\{f, \tau f, \tau^{2} f, \ldots\right\}$ is a $\tau$-admissible sequence. For other examples of admissible sequences we refer to [6].

Received by the editors October 4, 1965 and, in revised form, April 22, 1966. 
Throughout this paper $P=\left\{p_{0}, p_{1}, p_{2}, \ldots\right\}$ will denote an admissible sequence. We note that, if $\alpha \geqq 0, \alpha P$ is also an admissible sequence. Also, if $f \in L_{1}$ and $|f| \leqq \sum_{k=n}^{n^{\prime}} p_{k},\left(0 \leqq n \leqq n^{\prime}\right)$ then $|T f| \leqq \sum_{k=n+1}^{n^{\prime}+1} p_{k}$.

The following lemma was first given, in a slightly different form, in [4]. In the following we indicate an outline of its proof and refer the reader for a complete proof to [4], [11], and [12].

LeMma 1. For any $f \in L_{1}, \lim _{n \rightarrow \infty}\left(\tau^{n} f / S_{n} P\right)=0$ a.e. on the set $\left\{x \mid 0<S_{\infty} P(x)\right\}$.

Proof. Assume that $f \in L_{1}^{+}$and let, for a fixed $\varepsilon>0, E_{n}$ be the support of

$$
\left(\tau^{n} f-\varepsilon S_{n} P\right)^{+} .
$$

Then one can obtain that

$$
\varepsilon \sum_{n=1}^{\infty} \int_{E_{n}} p_{0}<\infty,
$$

from which the proof follows.

Let $E \in \mathscr{F}$. Associated with $\tau, P$, and $E$ define a possibly finite sequence

$$
A=\left\{a_{0}, a_{1}, a_{2}, \ldots\right\}
$$

of measurable functions as follows:

$$
a_{0}=x p_{0},
$$

$$
a_{n}=\chi\left[p_{n}-\sum_{k=1}^{n} \tau^{k} a_{n-k}\right], \quad n \geqq 1,
$$

where $\chi$ is the characteristic function of $E$. An induction argument shows that the functions $a_{n}$ are nonnegative. The sequence $A$ is infinite if it consists of $L_{1}$-functions. Otherwise it terminates with the first function which does not belong to $L_{1}$.

Definition 2. We let $\Omega_{E}(P)=\sum_{k} \int a_{k},\left(0 \leqq \Omega_{E}(P) \leqq \infty\right)$ where $\left\{a_{0}, a_{1}, \ldots\right\}$ is given by $\left({ }^{*}\right)$ and the summation is taken over the set of indices $k$ for which $a_{k}$ is defined. If $f \in L_{1}^{+}$we write $\Omega_{E}(f)$ instead of $\Omega_{E}\left(f_{\tau}\right)$.

We note that $\Omega_{E}(\alpha P)=\alpha \Omega_{E}(P)$ for any $\alpha \geqq 0, \Omega_{E}(f) \leqq\|f\|$ for any $f \in L_{1}^{+}$and $\Omega_{E}(P)=0$ if and only if $S_{\infty} P=0$ a.e. on $E$.

A maximal ergodic theorem. The following theorem, which will be fundamental for the rest of this paper, is an extension of an ergodic lemma [1] to admissible sequences. Since its proof is similar to the proof given in [1], here we give only an outline.

THEOREM 1. Let $E \in \mathscr{F}$ and consider two $\tau$-admissible sequences $P=\left\{p_{0}, p_{1}, \ldots\right\}$ 
and $Q=\left\{q_{0}, q_{1}, \ldots\right\}$. Then $\lim _{\sup _{n \rightarrow \infty}}\left(S_{n} P-S_{n} Q\right) \geqq 0$ a.e. on $E$ implies that $\Omega_{E}(P) \geqq \Omega_{E}(Q)$.

For the proof we need the following

LEMMA 2. Let $E \in \mathscr{F}$ and let $A^{\prime}=\left\{a_{0}^{\prime}, a_{1}^{\prime}, \ldots\right\}$ be a sequence of nonnegative measurable functions such that $a_{0}^{\prime} \leqq \chi p_{0}$ and if $a_{0}^{\prime}, a_{1}^{\prime}, \ldots, a_{n-1}^{\prime} \in L_{1}$ then

$$
a_{n}^{\prime} \leqq \chi\left(p_{n}-\sum_{k=1}^{n} \tau^{k} a_{n-k}^{\prime}\right)
$$

where $\chi$ is the characteristic function of $E$. Then $\int S_{\infty} A^{\prime} \leqq \Omega_{E}(P)$.

Proof. Assuming $\Omega_{E}(P)<\infty$ we can define

$$
b_{n}=p_{n}-\sum_{k=0}^{n} \tau^{k} a_{n-k}, \quad n \geqq 0
$$

An induction argument shows that a corresponding sequence

$$
b_{n}^{\prime}=p_{n}-\sum_{k=0}^{n} \tau^{k} a_{n-k}^{\prime}, \quad n \geqq 0
$$

is also defined and $\left(b_{n}^{\prime}-b_{n}\right)$ 's are nonnegative $L_{1}$-functions for all $n \geqq 0$. Now consider $G_{n}=\int\left[\left(S_{n} A-S_{n} A^{\prime}\right)-\left(b_{n}^{\prime}-b_{n}\right)\right]$. Then one obtains that $G_{0}=0$ and $G_{n} \leqq G_{n+1}$ for all $n \geqq 0$ which shows that $0 \leqq G_{n}$, or $\int S_{n} A^{\prime} \leqq \int S_{n} A$ for all $n \geqq 0$.

Proof of Theorem 1. Let $\chi$ be the characteristic function of $E$. Assuming $\Omega_{E}(P)<\infty$, we define a sequence $R=\left\{r_{0}, r_{1}, r_{2}, \ldots\right\}$ as follows:

$$
\begin{aligned}
& r_{0}=\chi p_{0} \wedge q_{0} \\
& r_{n}=\chi\left(p_{n}-\sum_{k=0}^{n-1} \tau^{n-k} r_{k}\right) \wedge\left(q_{0}-S_{n-1} R\right), \quad n \geqq 1 .
\end{aligned}
$$

Lemma 2 shows that these functions are in $L_{1}^{+}$and $\int S_{\infty} R \leqq \Omega_{E}(P)$. Using the hypotheses on $P$ and $Q$ one obtains that $\int S_{\infty} R=\chi q_{0}$, hence $\chi q_{0} \leqq \Omega_{E}(P)$.

Let $C=\left\{c_{0}, c_{1}, c_{2}, \ldots\right\}$ be the sequence associated with $\tau, Q$, and $E$, as defined by $\left(^{*}\right)$, so that $\Omega_{E}(Q)=\sum_{k} \int c_{k}$. In the previous paragraph we obtained that $\int c_{0} \leqq \Omega_{E}(P)$. An induction argument, analogous to that given in [1]; shows that, if $\int S_{n} C \leqq \Omega_{E}(P)$ then $\int S_{n+1} C \leqq \Omega_{E}(P)$, hence completes the proof of the theorem.

A ratio ergodic theorem for admissible sequences is a direct consequence of Theorem 1. 
TheOREM 2. Let $P$ and $Q$ be two $\tau$-admissible sequences and $E \in \mathscr{F}$. Then $\Omega_{E}(Q)<\infty$ implies that $\lim _{n \rightarrow \infty}\left(S_{n} Q / S_{n} P\right)$ exists (and is finite) a.e. on

$$
E^{\prime}=E \cap\left\{x \mid S_{\infty} P(x)>0\right\} \text {. }
$$

Before the proof we note that this theorem will only be used for the special case where $Q=f_{\imath}, f \in L_{1}^{+}$. The theorem is stated for a general admissible sequence $Q$ for reasons of symmetry, since this does not add any difficulty.

The following proof is analogous to that given in [3] for the proof of the ChaconOrnstein theorem.

Proof of Theorem 2. If $\lim \sup _{n \rightarrow \infty}\left(S_{n} Q / S_{n} P\right)$ is infinite a.e. on a nonnegligible subset $G$ of $E^{\prime}$, then Theorem 1 gives that $\Omega_{G}(Q) \geqq \alpha \Omega_{G}(P)$ for all $\alpha \geqq 0$. This is a contradiction, since $\Omega_{G}(Q)<\infty$ by Lemma 2 and $\Omega_{G}(P)>0$.

If $\lim _{n \rightarrow \infty}\left(S_{n} Q / S_{n} P\right)$ does not exist on a nonnegligible subset $H$ of $E^{\prime}$ then one can find two numbers $\alpha<\beta$ and an $H^{\prime} \subset H$ with $\mu\left(H^{\prime}\right)>0$ such that

$$
\liminf _{n \rightarrow \infty}\left(S_{n} Q / S_{n} P\right) \leqq \alpha<\beta \leqq \limsup _{n \rightarrow \infty}\left(S_{n} Q / S_{n} P\right)
$$

a.e. on $H^{\prime}$. By Theorem 1, this implies that $\Omega_{H^{\prime}}(Q) \geqq \beta \Omega_{H^{\prime}}(P)$ and

$$
\alpha \Omega_{H^{\prime}}(P) \geqq \Omega_{H^{\prime}}(Q)
$$

which is a contradiction.

Identification of the limit. A positive contraction $\tau$ induces a decomposition of $X$ into two parts of different characters. We state this result, which is due to Hopf [10] and Chacon [5] in the following form:

TheOREM 3. Let $\tau$ be a positive contraction on $L_{1}=L(X, \mathscr{F}, \mu)$. Then $X$ can be written as the union of two disjoint sets $D$ and $C$, called the dissipative and conservative parts respectively, with the following properties:

(i) For any $f \in L_{1}^{+}, S_{\infty}(f, \tau)<\infty$, a.e. on $D$.

(ii) For any $f \in L_{1}^{+}, S_{\infty}(f, \tau)=\infty$ or 0 a.e. on $C$.

(iii) If $f \in L_{1}$ and $f=0$ a.e. on $D$ then $\tau f=0$ a.e. on $D$.

(iv) If $f \in L_{1}^{+}$and $f=0$ a.e. on $D$ then $\|\tau f\|=\|f\|$.

Proof. Let $f \in L_{1}, f>0$ and

$$
\begin{aligned}
& C=\left\{x \mid\left(S_{\infty}(f, \tau)\right)(x)=\infty\right\}, \\
& D=\left\{x \mid\left(S_{\infty}(f, \tau)\right)(x)<\infty\right\},
\end{aligned}
$$

which are defined up to sets of measure zero. Then Theorem 2 implies that $D$ and $C$ have the properties (i) and (ii). 
Now assume that (iii) is not true. Then one can find a function $f \in L_{1}^{+}, f=0$ a.e. on $D$ and a nonnegligible subset $G$ of $D$ and two numbers $\alpha, \beta$, such that $0<\alpha<\tau f$ a.e. on $G$ and $S_{\infty}(f, \tau) \leqq \beta$ a.e. on $G$. Since $S_{\infty}(f, \tau)=\infty$ a.e. on the support of $f$, one can choose $n$ large enough so that $S_{n}(f, \tau) \geqq 2 \beta f / \alpha$ a.e. except on a set $H$ with $\int_{H} f \leqq \frac{1}{4} \alpha \mu(G)$. Then it follows that $S_{n+1}(f, \tau)>\beta$ on a nonnegligible subset of $G$, which is a contradiction and proves (iii).

Finally assume that (iv) is not true. Then there exists a function $f \in L_{1}^{+}, f=0$ a.e. on $D$ such that $\|f\|-\|\tau f\|=\lambda>0$. Now choose $n$ large enough so that $S_{n}(f, \tau) \geqq 2\|f\| f / \lambda$ a.e. except on a set $H$ with $\int_{H} f \leqq \frac{1}{4} \lambda$. Then

$$
\left\|S_{n}(f, \tau)\right\|-\left\|\tau S_{n}(f, \tau)\right\|>\|f\| .
$$

But this is a contradiction, since $\sum_{k=0}^{\infty}\left[\left\|\tau^{k} f\right\|-\left\|\tau^{k+1} f\right\|\right] \leqq\|f\|$.

Definition 3. A (measurable) subset $E$ of $X$ is invariant (with respect to $\tau$ ) if $f \in L_{1}$ and $f=0$ a.e. on $X-E$ imply that $\tau f=0$ a.e. on $X-E$.

Note that the previous theorem gives that $C$ is invariant.

The following two lemmas formulate the recurrence properties of the conservative part. Using these results we will obtain a simple interpretation of $\Omega_{E}(P)$ (Lemma 5).

LEMMA 3. Let $E$ be a (measurable) subset of $C$, the conservative part, and let $f \in L_{1}^{+}, f=0$ a.e. on $X-E$. Then $\Omega_{E}\left(\tau^{n} f\right)=\|f\|$ for all $n \geqq 0$.

Proof. For $n=0$ the assertion is trivial. We first show that $\Omega_{E}(\tau f)=\|f\|$.

Let $\chi$ and $\chi^{\prime}$ be the characteristic functions of $E$ and $X-E$ respectively and let $R(\tau f, \tau, E)=\left\{g_{0}, g_{1}, \ldots\right\}$ and $R^{\prime}(\tau f, \tau, E)=\left\{g_{0}^{\prime}, g_{1}^{\prime}, \ldots\right\}$ be the sequences defined as

$$
\begin{array}{ll}
g_{0}=\chi \tau f, & g_{0}^{\prime}=\chi^{\prime} \tau f, \\
g_{n}=\chi \tau g_{n-1}^{\prime}, & g_{n}^{\prime}=\chi^{\prime} \tau g_{n-1}^{\prime}, \quad n \geqq 1,
\end{array}
$$

and

$$
\tau_{E} f=S_{\infty}[R(\tau f, \tau, E)]
$$

It is easy to check that $\tau_{E}$ can be extended linearly to the $L_{1}$ space $L_{1}(E)$ of integrable functions with support in $E$ and be considered as a positive contraction on this space. Note that $\Omega_{E}(\tau f)=\left\|\tau_{E} f\right\|$. An induction argument shows that, for all $n \geqq 0, S_{n}(f, \tau) \leqq S_{n}\left(f, \tau_{E}\right)$ a.e. on $E$. Hence $\tau_{E}$ is conservative on $E$ and

$$
\Omega_{E}(\tau f)=\left\|\tau_{E} f\right\|=\|f\| .
$$

Now assume that $\Omega_{E}\left(\tau^{n} f\right)=\|f\|$ for $n=0,1, \ldots, N$. Then

$$
\begin{aligned}
\Omega_{E}\left(\tau^{N+1} f\right) & =\Omega_{E}\left(\tau \chi \tau^{N} f\right)+\Omega_{E}\left(\tau \chi^{\prime} \tau^{N} f\right)=\left\|\chi \tau^{N} f\right\|+\Omega_{E}\left(\chi^{\prime} \tau^{N} f\right) \\
& =\Omega_{E}\left(\tau^{N} f\right)=\|f\| .
\end{aligned}
$$

Here we use the fact that $\Omega_{E}(g)=\Omega_{E}(\tau g)$ if $g=0$ a.e. on $E$. 
Definition 4. Let $E$ be a subset of the conservative part $C$ and let $\mathscr{I}_{E}$ be the class of all invariant subsets of $C$ which contain $E$. Then the measure-theoretic intersection $I(E)$ of the elements of $\mathscr{I}_{E}$ is the smallest invariant set containing $E$.

Note that $I(E)$ is defined up to a set of measure zero and is an invariant set. If $g$ is an $L_{1}^{+}$function whose support is equal to $E$, then $S_{\infty}(g, \tau)=\infty$ a.e. on $I(E)$, and $S_{\infty}(g, \tau)=0$ a.e. on $X-I(E)$.

LEMMA 4. Let $E$ be a subset of $C$ and let $f$ be an $L_{1}^{+}$function which has support in $I(E)$. Then $\Omega_{E}(f)=\|f\|$.

Proof. First observe that if $0 \leqq f \leqq u, u \in L_{1}$ and $\Omega_{E}(u)=\|u\|$ then $\Omega_{E}(f)=\|f\|$. Now let $g \in L_{1}^{+}$and let the support of $g$ be equal to $E$. Then for any $\varepsilon>0$ there exists an $n$ such that $S_{n}(g, \tau) \geqq f$ a.e. except on a set $G$ with $\int_{G} f \geqq\|f\|-\varepsilon$. Since $\Omega_{E}\left(S_{n}(g, \tau)\right)=\left\|S_{n}(g, \tau)\right\|$, we then have $\Omega_{E}(f) \geqq\|f\|-\varepsilon$, which proves the lemma.

LEMMA 5. If $E$ is a subset of the conservative part $C$, then, for any $\tau$-admissible sequence $P$,

$$
\Omega_{E}(P)=\lim _{n \rightarrow \infty} \int_{I(E)} p_{n}
$$

Proof. First we have, directly from the definitions, that

$$
\Omega_{E}(P) \leqq \lim _{n \rightarrow \infty} \int_{I(E)} p_{n}
$$

Hence, if $\Omega_{E}(P)=\infty$ the proof is complete. Otherwise the previous lemma gives that $\Omega_{E}(P) \geqq \Omega_{E}\left(p_{n}^{\prime}\right)$ where $p_{n}^{\prime}=\chi_{I} p_{n} \in L_{1}^{+}, \chi_{I}$ being the characteristic function of $I(E)$. This implies the conclusion of the lemma.

LEMMA 6. The invariant subsets of $C$ form a $\sigma$-field $\mathscr{I}$ with respect to $C$.

Proof. The only nontrivial step of the proof is to show that if $I \subset C$ is invariant then $I^{\prime}=C-I$ is also invariant. Let $\tau_{I^{\prime}}$ be defined as in the proof of Lemma 3. If $I^{\prime}$ is not invariant one can find a function $f \in L_{1}^{+}, f=0$ a.e. on $X-I^{\prime}$ such that $\left\|\tau_{I^{\prime}} f\right\|<\|f\|$. But this contradicts the fact that $\tau_{I^{\prime}}$ is conservative.

LemMA 7. Let $\Omega_{C}(Q)<\infty$. Then the restriction of $h=\lim _{n \rightarrow \infty}\left(S_{n} Q / S_{n} P\right)$ to $C$ is an $\mathscr{I}$-measurable function.

Proof. Let $E_{\alpha}=\{x \mid h(x) \geqq \alpha\} \cap C$. If $I\left(E_{\alpha}\right) \cap\left(C-E_{\alpha}\right)$ has nonzero measure, then there exists an $\varepsilon>0$ such that $I\left(E_{\alpha}\right) \cap\left(C-E_{\alpha-\varepsilon}\right)=G$ has also nonzero measure. Let $H=I(G) \cap E_{\alpha}$. Then it follows that $I(H)=I(G)$, or $\Omega_{G}(\cdot)=\Omega_{H}(\cdot)$. Now on $H$, $\lim _{n \rightarrow \infty}\left(S_{n} Q / S_{n} P\right) \geqq \alpha$ which implies that $\Omega_{H}(Q) \geqq \alpha \Omega_{H}(P)$ and from an analogous 
consideration for $G$ one obtains that $\Omega_{G}(Q) \leqq(\alpha-\varepsilon) \Omega_{G}(P)$. But this is a contradiction and shows that $E_{\alpha} \in \mathscr{I}$.

A standard approximation procedure of measurable functions by simple functions leads to the following result.

Theorem 4. Let I be an invariant subset of $C, P$ and $Q$ be two admissible sequences, such that $\left(S_{\infty} P\right)>0$ a.e. on I and $\Omega_{I}(P)<\infty, \Omega_{I}(Q)<\infty$. Then

$$
\lim _{n \rightarrow \infty} \int_{I} q_{n}=\lim _{n \rightarrow \infty} \int_{I} h p_{n}
$$

with $h=\lim _{n \rightarrow \infty}\left(S_{n} Q / S_{n} P\right)$.

Corollary. If $\mu(C)<\infty, \Omega_{c}(Q)<\infty$ and $S_{\infty}(P)>0$ a.e. on $C$, then

$$
\lim _{n \rightarrow \infty}\left(S_{n} Q / S_{n} P\right)=\lim _{n \rightarrow \infty} \frac{E\left[q_{n} \mid \mathscr{I}\right]}{E\left[p_{n} \mid \mathscr{I}\right]} \text { a.e. on } C \text {. }
$$

Nonpositive operators. We now consider a general, not necessarily positive contraction $T$. The following result [8], due to Chacon and Krengel, shows that $T$ can always be dominated, in a certain sense, by a positive contraction $\tau$.

THEOREM 5. For every bounded linear operator $T: L_{1} \rightarrow L_{1}$ there is a unique bounded, linear and positive operator $\tau: L_{1} \rightarrow L_{1}$ such that

(i) $\|\tau\| \leqq\|T\|$.

(ii) For all $f \in L_{1},|T f| \leqq \tau|f|$.

(iii) If $f \in L_{1}^{+}$then $\tau f=\sup _{|g| \leqq f: g \in L_{1}}|T g|$.

Proof. Let $\mathscr{P}$ be the class of finite (measurable) partitions $\Pi=\left[E_{1}, \ldots, E_{n}\right]$ of $X$, partially ordered in the usual way. For any $\Pi=\left[E_{1}, \ldots, E_{n}\right] \in \mathscr{P}$ and $f \in L_{1}^{+}$let

$$
\tau_{\text {II }} f=\sum_{k=1}^{n}\left|T_{\chi_{k}} f\right|
$$

where $\chi_{k}$ is the characteristic function of $E_{k}, k=1, \ldots, n$. Also, let $\Pi_{n}$ be a sequence of nondecreasing partitions such that

$$
\lim _{n \rightarrow \infty}\left\|\tau_{\Pi_{n}} f\right\|=\sup _{\Pi \in \mathscr{P}}\left\|\tau_{\Pi} f\right\| .
$$

One then defines $\tau^{\prime} f=\lim _{n \rightarrow \infty} \tau_{\mathrm{M}_{n}} f$ (a.e.) and obtains the transformation $\tau$ of the theorem as the unique linear extension of $\tau^{\prime}$ to $L_{1}$. For further details we refer the reader to [8].

Definition 5. The operator $\tau$ as given by Theorem 5 , is called the linear modulus of $T$. 
For the rest of this paper $\tau$ will denote the linear modulus of $T$. The quantity $\Omega_{E}(\cdot)$ is defined, as before, in terms of $\tau$. We note that a sequence $P=\left\{p_{0}, p_{1}, \ldots\right\}$ is $T$-admissible if and only if it is $\tau$-admissible.

Definition 6. Two $L_{1}$ functions $f$ and $g$ are called equivalent (with respect to $T$ ), in notation $f \sim g$, if there exists a strictly positive $L_{1}$ function $F$, such that

$$
\limsup _{n \rightarrow \infty} \frac{\left|S_{n}(f-g, T)\right|}{S_{n}(F, \tau)}=0 \text { a.e. on } C
$$

where $C$ is the conservative part of $X$ with respect to $\tau$.

It is clear that this relation is actually an equivalence relation. We also note that, if $f \sim g$ then $f+h \sim g+h$ for any $h \in L_{1}$ and also that $T^{n} f \sim f$ for all $n \geqq 1$ by Lemma 1 .

DEFINITION 7. The nonnegative number

$$
M=M(f)=\inf _{g \sim f}\|g\|
$$

is the minimal norm of $f$ (with respect to $T$ ).

In terms of these concepts we first note the following

LEMMA 8. If $\Omega_{E}(|g|) \geqq a$ for all $g \sim f$ then for any $\lambda>0, \eta>0$ there exists an $h \sim f$ such that

$$
\|h\| \leqq M+\lambda \text { and } \int_{E}|h| \geqq a-\eta
$$

where $M$ is the minimal norm of $f$.

Proof. Let $0<\varepsilon<\min \left(\lambda, \frac{1}{4} \eta\right),\|g\| \leqq M+\varepsilon, g \sim f$ and consider

$$
\begin{aligned}
& \left\{\alpha_{0}, \alpha_{1}, \ldots\right\}=R(g, T, E), \\
& \left\{\beta_{0}, \beta_{1}, \ldots\right\}=R^{\prime}(g, T, E), \\
& \left\{\gamma_{0}, \gamma_{1}, \ldots\right\}=R(|g|, \tau, E), \\
& \left\{\delta_{0}, \delta_{1}, \ldots\right\}=R^{\prime}(|g|, \tau, E),
\end{aligned}
$$

where $R$ and $R^{\prime}$ are as defined in the proof of Lemma 3.

Since $\Omega_{E}(|g|)=\sum_{k=0}^{\infty}\left\|\gamma_{k}\right\| \geqq a$, there exists an $n$ such that $\sum_{k=0}^{n}\left\|\gamma_{k}\right\| \geqq a-\frac{1}{2} \eta$. Let $h=\sum_{k=0}^{n} \alpha_{k}+\beta_{n}$, which is equivalent to $f$. Then, by simple inductions we obtain that

and

$$
(M \leqq)\|h\| \leqq\|g\| \leqq M+\varepsilon
$$

$$
M \leqq \sum_{k=0}^{n}\left\|\alpha_{k}\right\|+\left\|\beta_{n}\right\| \leqq \sum_{k=0}^{n}\left\|\gamma_{k}\right\|+\left\|\delta_{n}\right\| \leqq M+\varepsilon
$$

and also $\left\|\beta_{n}\right\| \leqq\left\|\delta_{n}\right\|, \sum_{k=0}^{n}\left\|\alpha_{k}\right\| \leqq \sum_{k=0}^{n}\left\|\gamma_{k}\right\|$ which imply that

$$
\int_{E}|h|=\left\|\sum_{k=0}^{n} \alpha_{k}\right\| \geqq \sum_{k=0}^{n}\left\|\gamma_{k}\right\|-2 \varepsilon \geqq a-\eta .
$$


The following lemma shows that if the norm of an $L_{1}$-function $f$ is very close to its minimal norm, then the action of $T$ on this function can be described in terms of the action of $\tau$ on $|f|$.

LEMMA 9. Let $f \in L_{1}$ and let $M$ be its minimal norm. Then for any $\varepsilon>0$ there exists $a \delta>0$ with the following property. If $g \sim f$ and $\|g\| \leqq M+\delta$ then one can find $a$ function $\Delta \in L_{1}^{+}$with $\|\Delta\|<\varepsilon$ and a set $G$ with $\int_{G}|g|<\varepsilon$ such that, for any $n \geqq 0$,

$$
\sum_{k=0}^{n}\left|T^{k} g-e^{i \theta} \tau^{k}\right| g|| \leqq \sum_{k=0}^{n} \tau^{k} \Delta
$$

a.e. on $(S \cap C)-G$, where $S$ is the support of $g$ and $\theta: S \rightarrow(-\pi, \pi]$ is the phase of $g$.

The proof of this theorem, although quite straightforward, is rather long and will be divided into several sublemmas.

Let $\eta, 0<\eta<\pi$, and $\delta>0$ be fixed and $g \sim f,\|g\| \leqq M+\delta$. Define two sequences $R=\left\{r_{0}, r_{1}, r_{2}, \ldots\right\}$ and $R^{\prime}=\left\{r_{0}^{\prime}, r_{1}^{\prime}, r_{2}^{\prime}, \ldots\right\}$ of $L_{1}$ functions:

$$
\begin{array}{ll}
r_{0}=0, & r_{0}^{\prime}=g, \\
r_{n}=\lambda_{n} \operatorname{Tr}_{n-1}^{\prime}, & r_{n}^{\prime}=\left(1-\lambda_{n}\right) \operatorname{Tr}_{n-1}^{\prime}, \quad n \geqq 1,
\end{array}
$$

where $\lambda_{n}: X \rightarrow[0,1]$ for all $n \geqq 1$, such that $\lambda_{n}=0$ on the set on which $g=0$ or $\operatorname{Tr}_{n-1}^{\prime}=0$ or $\theta-\eta<\mathrm{ph} \operatorname{Tr}_{n-1}^{\prime}<\theta+\eta(\bmod 2 \pi)$, where "ph" denotes the phase of its argument. Outside of this set $\lambda_{n}$ is defined by the following (pointwise) relation $\lambda_{n}=\sup \left\{t \mid 0 \leqq t \leqq 1, \theta-\eta / 2 \leqq \operatorname{ph}\left[g+S_{n-1} R+t \operatorname{Tr}_{n-1}^{\prime}\right] \leqq \theta+\eta / 2(\bmod 2 \pi)\right\}$.

In what follows, if

$$
A=\left\{a_{0}, a_{1}, a_{2}, \ldots\right\}
$$

is a sequence of functions, $|A|$ and $\|A\|$ will denote the sequences

$$
\left\{\left|a_{0}\right|,\left|a_{1}\right|,\left|a_{2}\right|, \ldots\right\} \text { and }\left\{\left\|a_{0}\right\|,\left\|a_{1}\right\|,\left\|a_{2}\right\|, \ldots\right\}
$$

respectively.

LEMMA 10. $(1-\cos (\eta / 2)) S_{\infty}\|R\| \leqq 2 \delta$.

Proof. First, an induction argument shows that $S_{n}\|R\|+\left\|r_{n}^{\prime}\right\| \leqq M+\delta$. But we also have that $S_{n} R+r_{n}^{\prime} \sim g$, which combined with the first inequality, gives that

$$
\|g\|+S_{n}\|R\|-\left\|g+S_{n} R\right\| \leqq 2 \delta
$$

or

$$
\sum_{k=1}^{n}\left[\left\|g+S_{k-1} R\right\|+\left\|r_{k}\right\|-\left\|g+S_{k} R\right\|\right] \leqq 2 \delta
$$


Now, at almost every point on the support of $r_{k}$, the difference between the phases of $g+S_{k} R$ and $r_{k}$ is at least $\eta / 2$. Hence

$$
\left|g+S_{k} R\right| \leqq\left|g+S_{k-1} R\right|+\left|r_{k}\right| \cos \frac{\eta}{2}
$$

or

$$
\left(1-\cos \frac{\eta}{2}\right) S_{n}\|R\| \leqq 2 \delta
$$

for all $n \geqq 0$.

LEMMA 11. Let $G$ be the part of the support of $g$ on which $\mathrm{ph}\left(g+S_{n} R\right)=\theta-\eta / 2$ or $\theta+\eta / 2$ for at least one $n \geqq 0$. Then $(1-\cos (\eta / 2)) \int_{G}|g| \leqq 2 \delta$.

Proof. Write $G$ as the union of disjoint sets $G_{1}, G_{2}, \ldots$ such that on $G_{n}$ the phase of $g+S_{k} R$ is in the (open) interval $(\theta-\eta / 2, \theta+\eta / 2)$ for all $k=0,1, \ldots, n-1$, but the phase of $g+S_{n} R$ is $\theta-\eta / 2$ or $\theta+\eta / 2$.

Hence on $G_{n}$ :

$$
\left|g+S_{n} R\right| \leqq|g| \cos \frac{\eta}{2}+S_{n}|R|
$$

or

$$
\left(1-\cos \frac{\eta}{2}\right)|g| \leqq|g|+S_{n}|R|-\left|g+S_{n} R\right|=c_{n}
$$

where the last equality defines $c_{n}$.

From the proof of the previous lemma we have that $\int c_{n} \leqq 2 \delta$ for all $n \geqq 1$. Hence,

$$
2 \delta \geqq \int c_{n} \geqq \int_{\cup_{k=1}^{n} G_{k}} c_{n} \geqq \sum_{k=1}^{n} \int_{G_{k}} c_{k} \geqq\left(1-\cos \frac{\eta}{2}\right) \int_{\cup_{k=1}^{n} G_{k}}|g|,
$$

since $c_{n}$ is a nondecreasing sequence in $n$.

LEMMA 12. Let the sequence $H=\left\{h_{0}, h_{1}, \ldots\right\}$ be defined as $h_{0}=0$,

$$
h_{n}=\tau\left|r_{n-1}^{\prime}\right|-\left|\operatorname{Tr}_{n-1}^{\prime}\right|, \quad n \geqq 1 .
$$

Then

$$
S_{\infty}\|H\| \leqq \delta .
$$

Proof. First, an induction argument shows that

$$
\left\|\operatorname{Tr}_{k-1}^{\prime}\right\| \leqq\|g\|-S_{k-1}\|R\|-S_{k}\|H\|, \quad k \geqq 1 .
$$

Now

$$
S_{n-1} R+r_{n-1}^{\prime} \sim S_{n-1} R+\operatorname{Tr}_{n-1}^{\prime} \sim g .
$$


Hence

$$
\begin{aligned}
M & \leqq S_{n-1}\|R\|+\left\|\mathrm{Tr}_{n-1}^{\prime}\right\| \\
& \leqq\|g\|-S_{k}\|H\| \\
& \leqq M+\delta-S_{k}\|H\|
\end{aligned}
$$

which proves the lemma.

LEMMA 13. Let

$$
a_{n}=\tau^{n}|g|-\sum_{k=1}^{n} \tau^{n-k}\left|r_{k}\right|-\left|r_{n}^{\prime}\right|
$$

and

$$
b_{n}=e^{i \theta}\left[\tau^{n}|g|-\sum_{k=1}^{n} \tau^{n-k}\left|r_{k}\right|\right]-r_{n}^{\prime}
$$

Then

$$
a_{n}=\sum_{k=1}^{n} \tau^{n-k} h_{k}
$$

and

$$
\left|b_{n}\right| \leqq a_{n}+\eta \tau^{n}|g| \text { a.e. on }(C \cap S)-G \text {. }
$$

Proof. The first assertion follows directly from the definitions. For the second assertion, we first note that

$$
b_{n}=e^{i \theta}\left[\tau^{n}|g|-\sum_{k=1}^{n-1} \tau^{n-k}\left|r_{k}\right|\right]-\left[r_{n}^{\prime}+\left|r_{n}\right| e^{i \theta}\right] .
$$

On $(C \cap S)-G$ we have either $\lambda_{n}=0$ or $\lambda_{n}=1$. If $\lambda_{n}=0$ then $r_{n}=0$ and the phase of $r_{n}^{\prime}=\operatorname{Tr}_{n-1}^{\prime}$ is in the interval $(\theta-\eta, \theta+\eta)$. But $\left|r_{n}^{\prime}\right| \leqq \tau^{n}|g|$ and $\left|b_{n}\right| \leqq a_{n}+\eta \tau^{n}|g|$ follows. If $\lambda_{n}=1$ then $r_{n}^{\prime}=0$ and $\left|b_{n}\right|=a_{n}$.

Proof of Lemma 9. From the definitions and the previous lemma we have that

$$
\begin{aligned}
\left|e^{i \theta} \tau^{n}\right| g\left|-T^{n} g\right| & \leqq\left|b_{n}\right|+2 \sum_{k=1}^{n} \tau^{n-k}\left|r_{k}\right| \\
& \leqq \eta \tau^{n}|g|+\sum_{k=1}^{n} \tau^{n-k}\left(h_{k}+2\left|r_{k}\right|\right)
\end{aligned}
$$

Therefore

$$
\sum_{k=0}^{n}\left|e^{i \theta} \tau^{k}\right| g\left|-T^{k} g\right| \leqq \sum_{k=0}^{n} \tau^{k}\left[\eta|g|+S_{\infty}(H+2|R|)\right]
$$

Let

$$
\Delta=\eta|g|+S_{\infty}(H+2|R|)
$$


Then

$$
\|\Delta\| \leqq \eta\|g\|+\delta+\frac{4 \delta}{1-\cos \frac{\eta}{2}}
$$

Choose $\eta$, so that $M \eta \leqq \varepsilon / 4,0<\eta<\pi$ and choose $\delta$ satisfying

$$
0<\delta<\frac{\varepsilon}{16}\left(1-\cos \frac{\eta}{2}\right)
$$

Then it is easily seen that $\|\Delta\| \leqq \varepsilon$ and $\int_{G}|g|<\varepsilon$.

A general ergodic theorem. We now apply Lemmas 8 and 9 and the results for positive contractions to prove the following general ergodic theorem, due to Chacon [6], [7]. This theorem gives a mutual generalization of the Dunford-Schwartz theorem [9] and the Chacon-Ornstein theorem [4]. We note that Chacon's original paper [7] does not contain some details of the proof. A complete but rather complicated proof of the theorem appeared in [12].

THEOREM 6. For any $f \in L_{1}$,

$$
\lim _{n \rightarrow \infty}\left(S_{n}(f, T) / S_{n} P\right)
$$

exists (and is finite) a.e. on $\left\{x \mid 0<S_{\infty} P(x)\right\}$.

Proof. We can assume that $0<S_{\infty} P$ a.e. Since $\left|S_{n}(f, T)\right| \leqq S_{n}(|f|, \tau)$, it is also clear that lim $\sup _{n \rightarrow \infty}\left(\left|S_{n}(f, T)\right| / S_{n} P\right)$ is finite a.e. and that the limit (1) exists a.e. on $D$, the dissipative part of $\tau$. If it fails to exist on a nonnegligible subset of the conservative part $C$, then there exist a number $\alpha<0$ and a nonnegligible set $E \subset C$ such that

$$
\limsup _{n, m \rightarrow \infty}\left|\left(S_{n}(f, T) / S_{n} P\right)-\left(S_{m}(f, T) / S_{m} P\right)\right| \geqq \alpha \quad \text { a.e. on } E \text {. }
$$

We can also assume the existence of a number $\beta>0$ such that

$$
\limsup _{n \rightarrow \infty}\left(\left|S_{n}(f, T)\right| / S_{n} P\right) \leqq \beta \quad \text { a.e. on } E \text {. }
$$

Note that (2) and (3) are valid for any $g \sim f$.

Now (2) implies that, for any $g \sim f, \alpha / 2 \leqq \lim \sup _{n \rightarrow \infty}\left(S_{n}(|f|, \tau) / S_{n} P\right)$ a.e. on $E$. Hence, by Theorem 1,

$$
\Omega_{E}(|g|) \geqq \frac{1}{2} \alpha \Omega_{E}(P) \quad(>0)
$$

for any $g \sim f$, and by Lemma 8 , for any $\delta>0$ one can find a $g \sim f$ such that $\|g\| \leqq M+\delta$ and

$$
\int_{E}|g| \geqq \frac{1}{4} \alpha \Omega_{E}(P)
$$


Now let $\varepsilon>0$ be a fixed number and choose $\delta>0, g, \Delta, G$ as they are given by Lemma

9. We also assume that $g$ satisfies (4).

Let $F=(E-G) \cap S$ where $S$ is the support of $g$. Then, by Lemma 9,

$$
\sum_{k=0}^{n}\left|T^{k} g-e^{i \theta} \tau^{k}\right| g|| \leqq \sum_{k=0}^{n} \tau^{k} \Delta
$$

a.e. on $F$, with $\|\Delta\| \leqq \varepsilon$ and

$$
\int_{F}|g| \geqq \int_{E}|g|-\varepsilon \geqq \frac{1}{4} \alpha \Omega_{E}(P)-\varepsilon
$$

We now have, a.e. on $F$,

$$
\begin{aligned}
\beta & \geqq \limsup _{n \rightarrow \infty}\left|\frac{S_{n}(g, T)}{S_{n} P}\right| \\
& \geqq \lim _{n \rightarrow \infty} \frac{S_{n}(|g|, \tau)}{S_{n} P}\left[1-\limsup _{n \rightarrow \infty}\left|\frac{S_{n}(g, T)}{e^{i \theta} S_{n}(|g|, \tau)}-1\right|\right] \\
& \geqq \lim _{n \rightarrow \infty} \frac{S_{n}(|g|, \tau)}{S_{n} P}\left[1-\lim _{n \rightarrow \infty} \frac{S_{n}(\Delta, \tau)}{S_{n}(|g|, \tau)}\right] .
\end{aligned}
$$

Let

$$
l=\lim _{n \rightarrow \infty} \frac{S_{n}(\Delta, \tau)}{S_{n}(|g|, \tau)}
$$

Then, from Theorem 1 and Lemma 7 it follows that

$$
\int_{F} l|g| \leqq\|\Delta\| \leqq \varepsilon
$$

Since $l \geqq 0$, if $R=F \cap\left\{x \mid l(x) \leqq \frac{1}{2}\right\}$ then

$$
\frac{1}{2} \int_{F-R}|g| \leqq \varepsilon
$$

which, combined with (5) implies that

$$
\int_{R}|g| \geqq \frac{1}{4} \alpha \Omega_{E}(P)-3 \varepsilon .
$$

Now, by (6),

$$
\lim _{n \rightarrow \infty} \frac{S_{n}(|g|, \tau)}{S_{n} P} \leqq 2 \beta \quad \text { a.e. on } R
$$

hence

$$
\Omega_{R}(P) \geqq \frac{1}{2 \beta} \Omega_{R}(|g|) \geqq \frac{1}{2 \beta}\left[\frac{1}{4} \alpha \Omega_{E}(P)-3 \varepsilon\right]
$$


On the other hand, (2) implies that, a.e. on $R$,

$$
\begin{aligned}
\alpha & \leqq \limsup _{n, m \rightarrow \infty}\left|\frac{S_{n}(g, T)}{S_{n} P}-\frac{S_{m}(g, T)}{S_{m} P}\right| \\
& =\lim _{n \rightarrow \infty} \frac{S_{n}(|g|, \tau)}{S_{n} P} \limsup _{n, m \rightarrow \infty}\left|\frac{S_{n}(g, T)}{e^{i \theta} S_{n}(|g|, \tau)}-\frac{S_{m}(g, T)}{e^{i \theta} S_{m}(|g|, \tau)}\right| \\
& \leqq 2 \lim _{n \rightarrow \infty} \frac{S_{n}(|g|, \tau)}{S_{n} P} \lim _{n \rightarrow \infty} \frac{S_{n}(\Delta, \tau)}{S_{n}(|g|, \tau)}=2 \lim _{n \rightarrow \infty} \frac{S_{n}(\Delta, \tau)}{S_{n} P}
\end{aligned}
$$

Hence

$$
\Omega_{R}(P) \leqq \frac{2}{\alpha} \Omega_{R}(\Delta) \leqq \frac{2 \varepsilon}{\alpha}
$$

But, if $\varepsilon$ is sufficiently small, (7) and (8) are incompatible, which means that the assumption (2) cannot be true and completes the proof.

Acknowledgement. The author wishes to express his gratitude to Professor R. V. Chacon for very illuminating discussions on the subject.

\section{REFERENCES}

1. M. A. Akcoglu, An ergodic lemma, Proc. Amer. Math. Soc. 16 (1965), 388-392.

2. G. D. Birkhoff, Proof of the ergodic theorem, Proc. Nat. Acad. Sci. U.S.A. 17 (1931), 656-660.

3. A. Brunel, Sur un lemme ergodique voisin du lemme de Hopf, C.R. Acad Sci. Paris 256 (1963), 5481-5484.

4. R. V. Chacon and D. S. Ornstein, A general ergodic theorem, Illinois J. Math. 4 (1960), 153-160.

5. R. V. Chacon, Identification of the limit of operator averages, J. Math. Mech. 11 (1962), 961-968.

6. — Operator averages, Bull. Amer. Math. Soc. 68 (1962), 351-353.

7. - Convergence of operator averages, Symposium on Ergodic Theory, Tulane University, New Orleans, La., October, 1961.

8. R. V. Chacon and U. Krengel, Linear modulus of a linear operator, Proc. Amer. Math. Soc. 15 (1964), 553-559.

9. N. Dunford and J. T. Schwartz, Convergence almost everywhere of operator averages, J. Math. Mech. 5 (1956), 129-178.

10. E. Hopf, The general temporally discrete Markov process, J. Math. Mech. 3 (1954), 13-45.

11. - On the ergodic theorem for positive linear operators, J. Reine Angew. Math. 205 (1960), 101-106.

12. K. Jacobs, Lecture notes on ergodic theory, Aarhus University, Aarhus, Denmark, 1962-63.

UNIVERSITY OF TORONTO,

Toronto, Ontario, Canada 